\title{
A Generic Approach Towards Nanostructured Surfaces Based on Supramolecular Nanostamping on Reactive Polymer Coatings***
}

\author{
By Sarah Thévenet, Hsien-Yeh Chen, Jörg Lahann, and Francesco Stellacci*
}

Recent advances in biology and medicine have fuelled the need for smaller and more complex devices that offer increasing information densities at the low cost of a consumable. Based on organic and biological materials, a variety of parallel fabrication techniques that are suited for mass production of biodevices with high information densities are currently under development. Some methods, such as nanoimprinting, have outstanding spatial resolution but are mostly limited to expensive, inorganic materials. ${ }^{[1,2]}$ Others, like microcontact printing $(\mu \mathrm{CP})$, are compatible with both inorganic and organic materials, ${ }^{[3-5]}$ but although some of their variations have outstanding resolution ${ }^{[6-8]}$ and can print more than one type of molecule per stamping cycle, ${ }^{[5,8-13]}$ they still cannot handle the fabrication of complex high-resolution devices. The latter is an important limitation for biomedical devices, such as DNA microarrays, which often consist of as many as one million different DNA strands. ${ }^{[4]}$ These high-density arrays are currently produced with costly and laborious serial (spotting) or semiparallel (photolithography) methods and rely for the most part on in situ synthesis. ${ }^{[4]}$ Interestingly, all of the true parallel methods are based on contact between two surfaces.

Recently Crook's ${ }^{[14-16]}$ and our group ${ }^{[17-19]}$ independently developed a method that combines the high resolution capabilities of nanoimprinting with the soft material "spirit" of $\mu \mathrm{CP}$. In addition, our method can transfer patterns from a master surface to a secondary surface preserving their chemical nature, that is, two dots of different chemical composition can be stamped at the same time while maintaining their chemical differences. ${ }^{[9,11-13,15,20,21]}$ We refer to this property as chemical information transfer. To date, our method is the soft material stamping technique with the best spatial resolution $^{[19]}$ and among the only ones with proven chemical information transfer ability. ${ }^{[15,18]}$ We call our method supramolecular nanostamping (SuNS). It comprises three steps, shown in

[*] Prof. F. Stellacci, S. Thevenet Department of Materials Science and Engineering Massachusetts Institute of Technology 77 Massachusetts Avenue, Cambridge, MA 02139 (USA) E-mail: frstella@mit.edu

H.-Y. Chen, Prof. J. Lahann

Chemical Engineering Department University of Michigan

2300 Hayward Street, Ann Arbor, MI 48109 (USA)

[**; F.S. acknowledges the support of the Packard Foundation, the $3 \mathrm{M}$ untenured faculty award, and the DuPont Young Faculty Award
Scheme 1. An original master that consists of a patterned monolayer of single-stranded DNA (ssDNA) molecules is immersed in a solution of complementary DNA sequences (cDNA), suitably $5^{\prime}$ modified so to terminate with chemical groups that can bind to a surface. After hybridization the master is brought into contact with another substrate (replica). The functionalized cDNA's end binds to the replica. Upon heating the two substrates the DNA dehybridizes, allowing the two substrates to come apart. The master and replica obtained contain the same DNA pattern of complementary chemical structure. Crooks' group used force to separate the two substrates ${ }^{[14-16]}$ and has recently shown that cDNA can be synthesized directly on the master. ${ }^{[14]}$

Being a chemical-bond-based contact printing method, SuNS has the major disadvantage of needing a new immobilization protocol every time a new secondary substrate/binding group combination is selected. This is in stark contrast with the overall versatility and robustness of the process. Here we report a new approach to generalize SuNS to become an essentially substrate-independent stamping technique. The approach relies on functionalization of surfaces with reactive polymer coatings via chemical vapor deposition (CVD) polymerization. ${ }^{[22]}$ CVD polymerization has been used to modify a wide range of different substrates with a series of different functional groups, including amines, ${ }^{[23]}$ active esters, ${ }^{[24]}$ anhydrides, ${ }^{[25]}$ alkynes, ${ }^{[26]}$ aldehydes, ${ }^{[27]}$ or ketones. $^{[28]}$

Using a modified Gorham process, the CVD polymerization uses substituted [2.2]paracyclophanes as starting materials and results in ultrathin, conformal polymer coatings. CVD modified substrates have been used for immobilization of proteins or sugars and have been spatially modified by $\mu \mathrm{CP}$ as well as photolithographic methods in the past. ${ }^{[29]}$ This simple, one-step surface modification, in addition to being substrateindependent, offers accurate control of the chemical composition and the architecture of the films, and an excellent adhesion. Because the reactive coatings can exhibit many chemically reactive groups, cDNA can be tethered to the surface in a variety of manners. In the scope of this Communication we focused on one chemistry for the attachment of DNA, using poly(4-formyl-p-xylylene-co-p-xylylene) that links to $5^{\prime}$ amine modified cDNA by forming an imine bond. The aldehydefunctionalized reactive coating was deposited on glass, silicon, poly(dimethylsiloxane) (PDMS), poly(methyl methacrylate) (PMMA), and polystyrene (PS), as previously reported. ${ }^{[20]}$ The chemical structure of the polymer coating has been confirmed by X-ray photoelectron spectroscopy (XPS) and graz- 


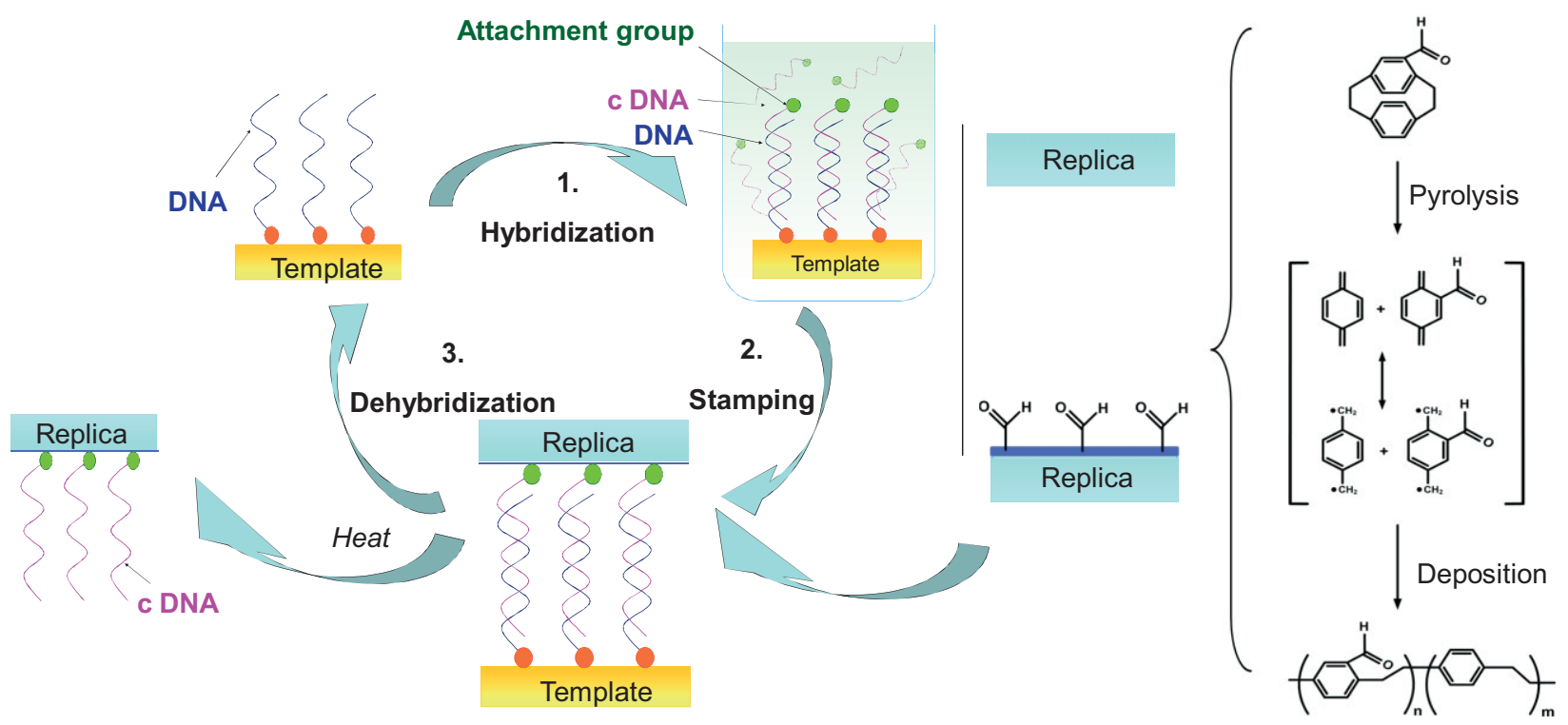

Scheme 1. Schematic illustration of the supramolecular nanostamping (SuNS) process. The scheme on the right shows the approach used to achieve a substrate-independent stamping technique.

ing angle Fourier transformed infrared spectroscopy (FTIR) to be i) independent of the substrate used, and ii) in agreement with the previously reported data. ${ }^{[27]}$ Specifically, the presence of aldehyde groups was confirmed by FTIR stretches at 1688 and $2734 \mathrm{~cm}^{-1}$ and a signal at $287.9 \mathrm{eV}$ in the high-resolution C 1s spectrum. The poly(4-formyl- $p$-xylylene-co- $p$-xylylene) coatings were $50-70 \mathrm{~nm}$ thick, as determined by ellipsometry.

To prove that different substrates coated with the same polymer could be stamped on with the same approach we prepared masters consisting of evenly spaced ssDNA lines. The masters were prepared starting from a silicon substrate made using either interference lithography or achromatic interference lithography. In the former case the line pitch was $740 \mathrm{~nm}$ with $370 \mathrm{~nm}$ thick silicon oxide lines. In the latter case the pattern pitch was $100 \mathrm{~nm}$ with $50 \mathrm{~nm}$ silicon oxide lines. The top part of the lines was gold coated through shadow evaporation (see Experimental). A ssDNA (5'modified with hexyl-thiol) monolayer was assembled on the gold layer by placing the master in a $4 \mu \mathrm{M}$ DNA solution for 4 days. Throughout this Communication, DNA strands composed of 50 bases were used. These masters were used to stamp onto silicon, quartz, PS, PMMA, and PDMS, all coated with a layer of poly(4-formyl- $p$-xylylene-co-p-xylylene). Typical results are shown in Figure 1. Briefly, our stamping procedure consisted of placing a drop of $1 \mu \mathrm{M} 5^{\prime}$ hexylamine modified cDNA solution onto the master in a humid chamber for at least two hours. After this period, the master was cleaned and dried, and the polymer coated replica, without any additional pretreatment, was brought into contact with the master. A slight pressure was applied and maintained overnight to the substrates, which were kept in a vacuum desiccator. The two substrates were then heated at $90{ }^{\circ} \mathrm{C}$ to dehybridize the DNA strands, and sep-
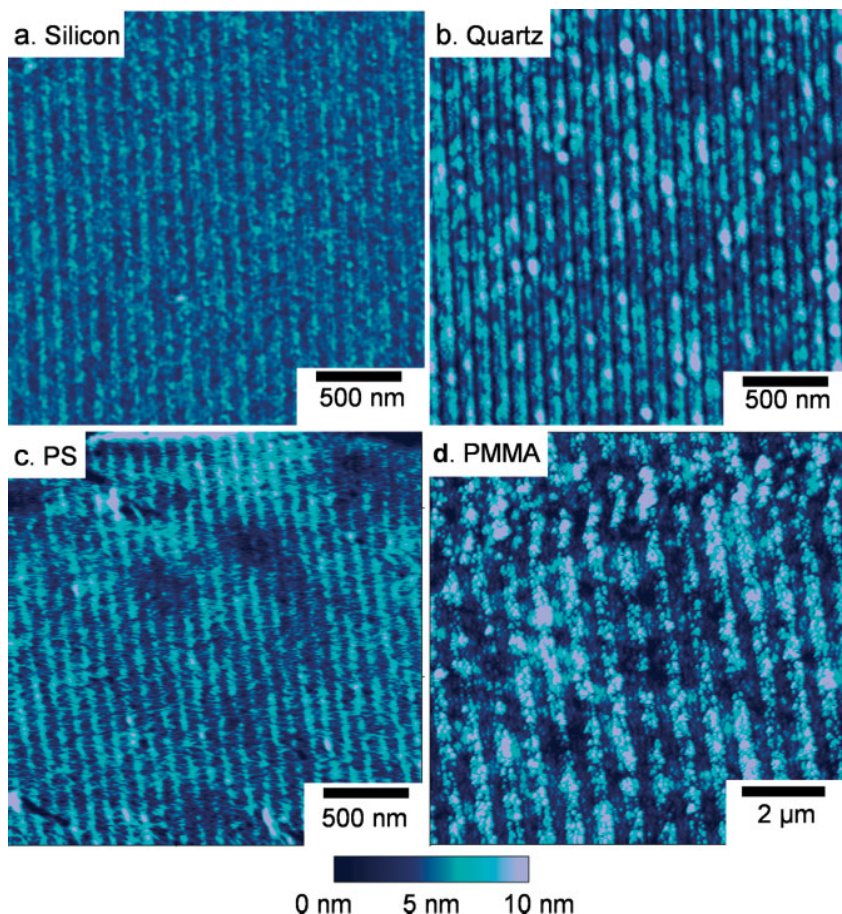

Figure 1. Tapping mode AFM images of DNA lines stamped on a variety of substrates. a) silicon; b) quartz; c) polystyrene (PS); d) poly(methylmethacrylate) (PMMA). The masters used for these printings are gold coated silicon grating patterns with a $100 \mathrm{~nm}$ pitch $(\mathrm{a}-\mathrm{c})$ and a $700 \mathrm{~nm}$ pitch (d). For this set of experiments masters have been used to stamp successively on as many as 7 different substrates.

arated. Detailed procedures are described in the Experimental section. The printed substrates were imaged using atomic force microscopy (AFM) in tapping mode (Fig. 1), parallel 
lines with the correct pitch and with thicknesses varying from $100 \mathrm{~nm}$ to $390 \mathrm{~nm}$ and from $25 \mathrm{~nm}$ to $75 \mathrm{~nm}$ were found when printing from the $740 \mathrm{~nm}$ pitch and the $100 \mathrm{~nm}$ pitch masters, respectively. The thickness seemed to vary because of printing conditions (mostly pressure applied) but not because of the substrate used. The printed DNA lines had a height between 3 and $10 \mathrm{~nm}$, showing that the printed DNA lines are made of monolayers not dense enough to force the DNA to stand (in that case the lines would be $\sim 20 \mathrm{~nm}$ tall) but also not sparse enough to cause DNA to lay on the substrate (in that case a height of 1 to $2 \mathrm{~nm}$ would have been observed). ${ }^{[30]}$ Again, the height of the DNA strands appeared to be independent of the substrate material.

In order to prove that the lines observed in the AFM images were actually made of ssDNA of a sequence complementary to the original one, we employed a unique characteristic (and advantage) of SuNS: the possibility of reusing a printed replica as master. Only if the stamping process has worked correctly, the printed substrate can be "inked" with DNA of the original sequence (suitably $5^{\prime}$ modified) and reused as master. Specifically, we used one of the printed silicon substrates to print onto poly(4-formyl- $p$-xylylene-co- $p$-xylylene)coated PS using the procedure described above. The results are shown in Figure 2. The high pattern fidelity observed in Figure 2 corroborates our model, in that the stamped pattern consists of DNA strands of the expected sequence in a conformation that retains binding activity.
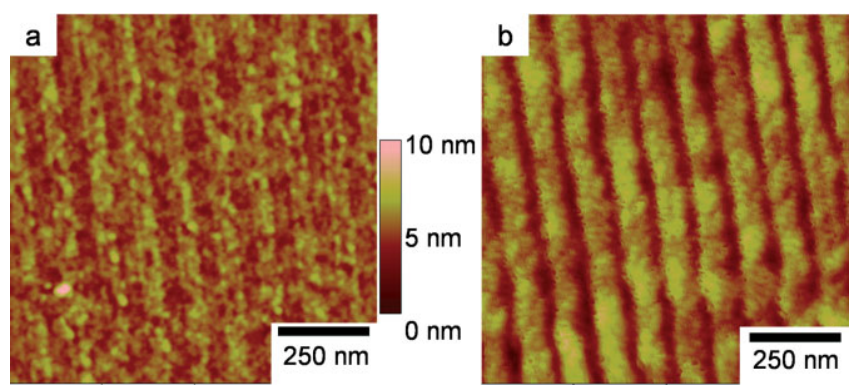

Figure 2. Substrates printed using a printed substrate as a master. a) Tapping mode AFM image of a DNA-printed poly(4-formyl-p-xylyleneco-p-xylylene)-coated silicon substrate used as a master. b) Tapping mode AFM image of a poly (4-formyl-p-xylylene-co-p-xylylene)-coated polystyrene substrate reprinted from the printed silicon substrate shown in (a).

Further control experiments aiming at demonstrating that the observed AFM patterns were made of ssDNA of the correct sequence were performed using fluorescence microscopy. The masters used in these experiments were silicon substrates photolithographically patterned with $1 \mu \mathrm{m}$ large gold squares, on which a monolayer of thiolated ssDNA was assembled. These masters were used to print onto poly(4-formyl- $p$-xylylene-co-p-xylylene)-coated PS and PDMS substrates using the above-mentioned protocol. The stamped substrates were placed in a solution of fluorescently labeled DNA strands of the same sequence as the one present on the master. The hy- bridized substrates were then imaged with a fluorescence microscope. As shown in Figure $3 \mathrm{a}-\mathrm{b}$, the correct pattern of squares could be imaged using fluorescence microscopy. Furthermore, the same sample was re-imaged after a dehybri-

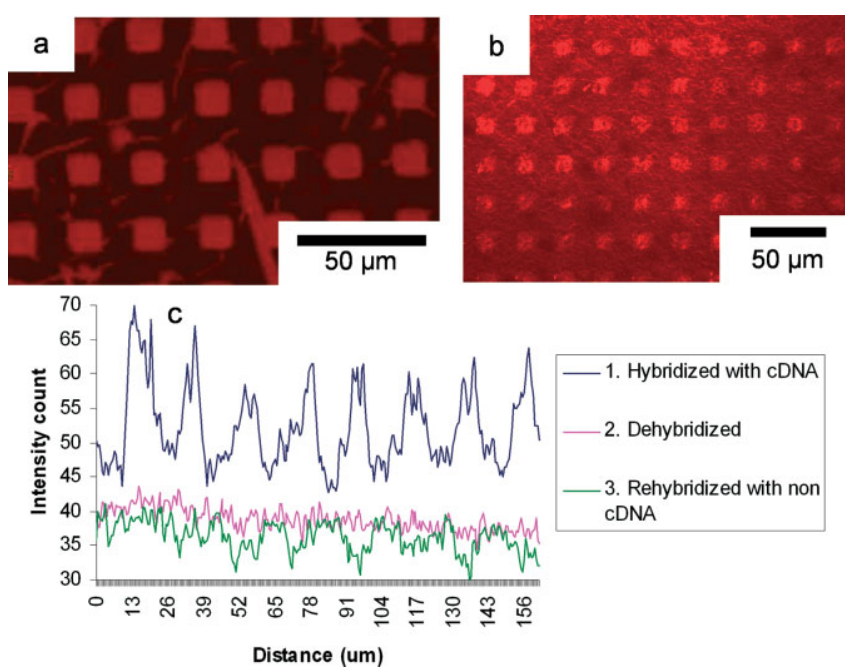

Figure 3. Fluorescence microscope image of a) a printed PDMS substrate, b) a printed PS substrate. After their stamping the samples were hybridized with fluorescently labeled DNA strands before being imaged. c) Line scans of fluorescence microscopy images obtained (blue) after hybridizing the sample to fluorescently labeled complementary DNA strands, (pink) after heating such sample so to induce dehybridization, and (green) after exposing the sample to non complementary fluorescently labeled DNA.

dization step, in which the substrate was immersed and cleaned in a hot water solution. The line plot in Figure $3 b$ shows that the contrast along one imaging line disappeared after this process. To further prove that the DNA present on the square was of the correct sequence we placed the printed substrate in a $1 \mu \mathrm{M}$ solution of fluorescently labeled ssDNA of a sequence that was not complementary to the one originally stamped. As expected in this case, no contrast was observed on the imaged (see Fig. 3c, green line). These experiments clearly prove that only ssDNA of the programmed sequence was printed on the poly(4-formyl- $p$-xylylene-co- $p$-xylylene)modified substrates.

Because the binding chemistry was the same for all the substrates, SuNS's protocols were nearly identical for all the substrates. The only difference was the method used to provide contact between the master and the secondary substrate during the stamping step itself. The substrate's mechanical properties (mainly rigidity) drastically change the way pressure is transferred into good surface-to-surface contact. Hard substrates like silicon and quartz were stamped simply using a mechanical vise adapted with soft PDMS plates. This sometimes leads to overall contact areas limited by the presence of macroscopic roughness or dust particles. Polymeric substrates offer the unique property of allowing for softening with the consequent improvement of conformity to the substrate they 
are pushed onto. Hence at the beginning of the stamping step we elevated the temperature to $60^{\circ} \mathrm{C}$, enabling the secondary polymeric substrate to conform better to the intrinsic roughness of the master's surface, thus increasing the printed area. As for a soft material like PDMS, we gently applied uniform pressure by inflating a balloon placed between the substrate and the upper part of our mechanical vice. As it is inflated, the balloon applies a growing pressure on the substrates starting from the center of the substrates, therefore avoiding the trapping of air bubbles between the samples.

To demonstrate the versatility of this approach, we extended the technique to pre-patterned substrates. Because of the excellent conformal coatings obtained with chemical vapor deposition, reactive coatings can be deposited within confined geometries. ${ }^{[31]}$ We recently extended this approach to the facile fabrication of both continuous and discontinuous patterns using vapor-assisted micropatterning in replica structures. ${ }^{[32]}$ In the context of this study, combination with a secondary SuNS printing step can be used to generate hierarchical structures on different length scales. To prove this point, we printed $100 \mathrm{~nm}$ pitch DNA lines onto an array of $200 \mu \mathrm{m}$ squares spaced $200 \mu \mathrm{m}$, obtaining a superimposition of the two patterns as shown in Figure 4. PS substrates were coated with poly(4-formyl- $p$-xylylene-co- $p$-xylylene) as a square array with a periodicity of $400 \mu \mathrm{m}$. Such a prepatterned surface was used as a replica surface for SuNS starting with a $100 \mathrm{~nm}$

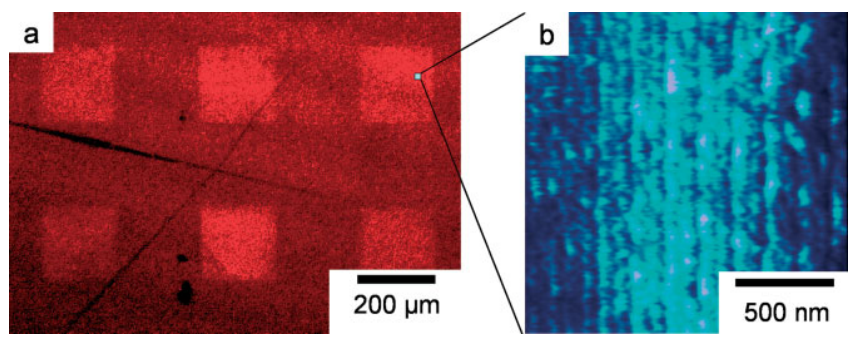

Figure 4. Hierarchical printing. a) Fluorescence microscopy image of a printed prepatterned polystyrene substrate. b) AFM image of the printed pattern inside a square.

pitch grating. The stamped surface was hybridized with its fluorescent modified complement. Fluorescence microscopy enabled us to check that the DNA was transferred only in the coated squares (Figure 4a), while using atomic force microscopy we could observe the printed patterns in the coated squares (Fig. 4b) and verify the absence of nanopatterns outside the coated squares. These data also clearly eliminate the possibility of imprinting (mechanical deformation ${ }^{[1]}$ ) as the cause of pattern formation.

In conclusion, we have shown that various surfaces can successfully be coated with reactive polymer coatings and then modified with DNA features using SuNS. This approach represents an important step forward, because it essentially renders SuNS a substrate-independent method. Indeed, it enabled us to print with the same protocol onto five very different materials: silicon, quartz, polystyrene, acrylic, and PDMS. Future work may include the extension of this method to reactive coatings with different functional groups or the employment of multi-functional coatings that offer two or more functional groups in defined surface ratios. ${ }^{[33]}$ With its potential for mass production, this new approach could benefit to a broad spectrum of applications in the field of bio- and nanodevices, where the development of inexpensive, consumable platforms that, at the same time, offer high information densities might have a profound impact.

\section{Experimental}

The substrates used were usually about $0.25-0.5 \mathrm{~cm}^{2}$ in size.

CVD Polymerization: Poly(4-formyl- $p$-xylylene-co-p-xylylene) was synthesized via CVD polymerization using a self-designed CVD installation. A defined amount $(100 \mathrm{mg})$ of the starting material, 4-formyl[2,2]paracyclophane, was sublimed under vacuum and converted by pyrolysis into the corresponding quinodimethanes, which spontaneously polymerized upon condensation to the substrate surface. A defined argon flow of $20 \mathrm{sccm}$ was used as carrier. Sublimation temperatures were kept between $95^{\circ} \mathrm{C}$ and $105^{\circ} \mathrm{C}$ followed by pyrolysis at $700^{\circ} \mathrm{C}$. Subsequently, polymerization occurred on a rotating, cooled sample holder $\left(25^{\circ} \mathrm{C}\right)$ placed inside a stainless steel chamber with a wall temperature of $120^{\circ} \mathrm{C}$. The sample was either homogeneously coated or patterned by selectively blocking certain surface areas with a PDMS replica structure. ${ }^{[32]}$ The coating pressure was 0.12 Torr $\left(1\right.$ Torr $\left.=1.333 \times 10^{2} \mathrm{~Pa}\right)$ or below. The exit of the chamber was connected to a mechanical pump via a cooling trap.

Fabrication of the Masters: The gratings were fabricated in the NanoStructures Laboratory at MIT. The $100 \mathrm{~nm}$ pitch gratings were obtained using achromatic interference lithography (AIL). The pattern was then transferred into silicon by reactive-ion etching. The silicon grating was then coated, by e-beam deposition, with $3 \mathrm{~nm}$ of titanium followed by $7 \mathrm{~nm}$ of gold. The $700 \mathrm{~nm}$ pitch silicon gratings were made using interference lithography, then coated by e-beam evaporation with $5 \mathrm{~nm}$ of chromium followed by $5 \mathrm{~nm}$ of gold.

The square patterns were obtained from silicon substrates by photolithography, followed by the deposition of a $5 \mathrm{~nm}$ adhesion layer of chromium followed by $25 \mathrm{~nm}$ of gold, by e-beam evaporation.

$D N A$ : DNA was purchased from Integrated DNA Technologies with commercially available $5^{\prime}$ modifications. Sequences used:

A: 5'- TCC CAA AGA ACA GTG GTG GCT CAA GCT ACG GCC CCT CAT GAA AAT CCT GG - $3^{\prime}$

Complementary sequence $A^{\prime}: 5^{\prime}$ - CCA GGA TTT TCA TGA GGG GCC GTA GCT TGA GCC ACC ACT GTT CTT TGG GA -3'

Non-complementary sequence B: $5^{\prime}$-TGA CTG CTG TAG TTC AGA AGA GGT TTG GCT TTC CAG AGG GCA GTG TAG AG -3'

DNA Immobilization on Gold: The gold substrate was soaked 4 days in a solution of $5^{\prime}$ hexyl-thiol modified 50mer single-stranded DNA of sequence A ( $4 \mu \mathrm{M}$ in $0.5 \mathrm{M}$ potassium phosphate buffer). It was then rinsed with Millipore water, immersed for $1 \mathrm{~h}$ in a $1 \mathrm{mM}$ 6-mercapto-1-hexanol aqueous solution to limit nonspecific adsorption of DNA during subsequent manipulations, rinsed again abundantly, and dried with a dust remover.

DNA Hybridization: A few microliters of a $1 \mu \mathrm{M}$ solution of $5^{\prime}$ hexylamine modified single-stranded complementary DNA (sequence $\mathrm{A}^{\prime}$ ) in hybridization buffer were dropped on the substrate and covered with a glass cover-slip. The hybridization buffer was $1 \mathrm{M} \mathrm{NaCl}$ in TE buffer for hybridization on gold, or $4 \times$ silane-sodium citrate (SSC), $0.1 \%$ sodium dodecyl sulfate (SDS) for hybridization on polymer coated substrates. The substrate was then left for a minimum of $2 \mathrm{~h}$ up to overnight in a sealed humid chamber. It was subsequently rinsed with $4 \times$ SSC buffer, diluted twice, followed by a brief rinse in Millipore water, and dried with a dust remover. 
DNA Dehybridization: The substrate was soaked twice 15 minutes in Millipore water at $90^{\circ}$ Cand rinsed abundantly.

General SuNS procedure: The patterned gold master was prepared as described above for the immobilization of thiol modified ssDNA of sequence $\mathrm{A}$, followed by hybridization with complementary amineterminated ssDNA (sequence $\mathrm{A}^{\prime}$ ). The poly(4-formyl- $p$-xylylene-co$p$-xylylene)-modified secondary substrate, dry and without any additional treatment, was then brought into contact with the dried master, and a slight pressure was applied and maintained overnight while the substrates are kept in a vacuum desiccator. The two substrates, while still under pressure, were subsequently placed in an oven at $90{ }^{\circ} \mathrm{C}$ for 30 min to dehybridize the DNA strands. The pressure was then released, and a droplet of $0.1 \mathrm{M} \mathrm{NaCl}$ in TE buffer at $90^{\circ} \mathrm{C}$ was put on the coupled samples to help them to come apart. The samples were then rinsed with Millipore water and blown dry with a dust remover.

Characterization: Tapping Mode AFM images were obtained using a Digital Instrument MulitMode Nanoscope IIIa, using a J scanner. All experiments presented in this Communication were performed using Veeco Nanoprobe tips (Model \#: RTESP, length: $125 \mu \mathrm{m}$, resonance frequency $\sim 300 \mathrm{kHz}$ ). Fluorescence microscopy images were obtained using a Zeiss Axioplan 2 with a mercury lamp. The imaging was performed using Rhodamine Red modified oligonucleotides, and the filters used in excitation and collection had maximum transmittance at $546 \mathrm{~nm}$ and $590 \mathrm{~nm}$, respectively.

Received: June 15, 2007

Revised: August 23, 2007

Published online: November 21, 2007

[1] S. Y. Chou, P. R. Krauss, W. Zhang, L. Guo, L. Zhuang, J. Vac. Sci. Technol., B 1997, 15, 2897.

[2] M. Colburn, S. C. Johnson, M. D. Stewart, S. Damle, T. C. Bailey, B. Choi, M. Wedlake, T. B. Michaelson, S. V. Sreenivasan, J. G. Ekerdt, C. G. Willson, Proc. SPIE 1999, 3676, 379.

[3] Y. N. Xia, G. M. Whitesides, Angew. Chem. Int. Ed. 1998, 37, 551.

[4] D. Gershon, Nat. Methods 2004, 1, 263.

[5] V. Mahalingam, S. Onclin, M. Peter, B. J. Ravoo, J. Huskens, D. N. Reinhoudt, Langmuir 2004, 20, 11756.

[6] J. P. Renault, A. Bernard, A. Bietsch, B. Michel, H. R. Bosshard, E. Delamarche, M. Kreiter, B. Hecht, U. P. Wild, J. Phys. Chem. B 2003, 107, 703 .

[7] B. Michel, A. Bernard, A. Bietsch, E. Delamarche, M. Geissler, D. Juncker, H. Kind, J. P. Renault, H. Rothuizen, H. Schmid, P. Schmidt-Winkel, R. Stutz, H. Wolf, IBM J. Res. Dev. 2001, 45, 697.
[8] C. M. Bruinink, C. A. Nijhuis, M. Peter, B. Dordi, O. Crespo-Biel, T. Auletta, A. Mulder, H. Schonherr, G. J. Vancso, J. Huskens, D. N. Reinhoudt, Chem. Eur. J. 2005, 11, 3988.

[9] A. Bernard, D. Fitzli, P. Sonderegger, E. Delamarche, B. Michel, H. R. Bosshard, H. Biebuyck, Nat. Biotechnol. 2001, 19, 866.

[10] J. P. Renault, A. Bernard, D. Juncker, B. Michel, H. R. Bosshard, E. Delamarche, Angew. Chem. Int. Ed. 2002, 41, 2320.

[11] S. A. Lange, V. Benes, D. P. Kern, J. K. H. Horber, A. Bernard, Anal. Chem. 2004, 76, 1641.

[12] A. Bernard, J. P. Renault, B. Michel, H. R. Bosshard, E. Delamarche, Adv. Mater. 2000, 12, 1067

[13] C. Thibault, V. Le Berre, S. Casimirius, E. Trévisiol, J. François, C. Vieu, J. Nanobiotech. 2005, $3,7$.

[14] J. Kim, R. M. Crooks, J. Am. Chem. Soc. 2006, 128, 12076.

[15] H. H. Lin, J. Kim, L. Sun, R. M. Crooks, J. Am. Chem. Soc. 2006, 128,3268 .

[16] H. H. Lin, L. Sun, R. M. Crooks, J. Am. Chem. Soc. 2005, 127, 11210.

[17] A. A. Yu, F. Stellacci, J. Mater. Chem. 2006, 16, 2868.

[18] A. A. Yu, T. A. Savas, G. S. Taylor, A. Guiseppe-Elie, H. I. Smith, F. Stellacci, Nano Lett. 2005, 5, 1061.

[19] A. A. Yu, T. Savas, S. Cabrini, E. diFabrizio, H. I. Smith, F. Stellacci, J. Am. Chem. Soc. 2005, 127, 16774.

[20] A. A. Yu, T. A. Savas, G. S. Taylor, A. Guiseppe-Elie, H. I. Smith, F. Stellacci, Nano Lett. 2005, 5, 1061.

[21] J. P. Renault, A. Bernard, D. Juncker, B. Michel, H. R. Bosshard, E. Delamarche, Angew. Chem. Int. Edit. 2002, 41, 2320.

[22] J. Lahann, Polym. Int. 2006, 55, 1361.

[23] J. Lahann, H. Höcker, R. Langer, Angew. Chem. Int. Ed. 2001, 40, 726.

[24] J. Lahann, I. S. Choi, J. Lee, K. F. Jensen, R. Langer, Angew. Chem. Int. Ed. 2001, 40, 3166.

[25] J. Lahann, D. Klee, H. Höcker, Macromol. Rapid Commun. 1998, 19, 441.

[26] H. Nandivada, H.-Y. Chen, L. Bondarenko, J. Lahann, Angew. Chem. Int. Ed. 2006, 45, 3360.

[27] H. Nandivada, H.-Y. Chen, J. Lahann, Macromol. Rapid Commun. 2005, 26, 1794.

[28] K. Y. Suh, R. Langer, J. Lahann, Adv. Mater. 2004, 16, 1401.

[29] H. Y. Chen, J. Lahann, Anal. Chem. 2005, 77, 6909.

[30] O. Akbulut, J.-M. Jung, R. Bennett, Y. Hu, R. Cohen, A. M. Mayes, F. Stellacci, unpublished.

[31] H. Y. Chen, Y. Elkasabi, J. Lahann, J. Am. Chem. Soc. 2006, 128, 374.

[32] H. Y. Chen, J. Lahann, unpublished.

[33] Y. Elkasabi, H. Y. Chen, J. Lahann, Adv. Mater. 2006, 18, 1521. 